\title{
READING SKILLS IN INDIVIDUALS WITH MACULAR DEGENERATION
}

\begin{abstract}
Macular degeneration (MD) involves a whole range of progressive and degenerative changes in the macula. Though central vision is affected, peripheral vision usually remains preserved in $M D$. Unfortunately, there is a lack of effective medical treatment for most $M D$, therefore it is important to focus efforts on the development of visual compensatory mechanisms.

People with macular degeneration experience difficulties in reading due to central-field loss. Reading skills is the primary difficulty and at the same time the primary goal for individuals with macular degeneration seeking vision rehabilitation. The current prevalent methods for reading skills training are magnificationlarge print size and scotoma awareness training. Also, more recent studies showed that specific oculomotor training, avoiding visual crowding and special fonts are all beneficial to reading skills rehabilitation. The focus of this article is to review and discuss the methods and adaptations available today that we can use in rehabilitation of reading skills in individuals with $M D$.
\end{abstract}

Keywords: MACULAR DEGENERATION, READING, ECCENTRIC VIEWING, FONTS

\section{Introduction}

Macular degeneration (MD) involves a whole range of progressive and degenerative changes in the macula. People with MD usually develop a central scotoma, which is an area of absent vision within the central visual field (absolute scotoma) or of diminished light perception (relative scotoma). Central scotoma affects visual acuity, contrast sensitivity and stereoscopic vision. Though central vision is affected, peripheral vision usually remains preserved in MD. In later stages, it severely impairs visual acuity, and therefore, many activities, e.g. reading, watching television, and driving becomes difficult or may even be impossible.

The most common form of MD is age-related macular degeneration (AMD), but there is also a juvenile form in younger people (JMD). Macular degeneration continues to be a leading cause of severe visual impairment in developed countries - AMD is expected to affect 288 million people worldwide by 2040 (Wong et al., 2014). Moreover, it has been estimated that one in three 
individuals older than 75 years and one in 30 individuals older 52 years were affected by AMD (Klein et al., 1997). Risk factors for developing AMD include aging, genetic heredity, smoking, and obesity (Jager, Mieler, \& Miller, 2008).

Unfortunately, there is a lack of effective medical treatment for most $\mathrm{MD}$, therefore it is important to focus efforts on the development of visual compensatory mechanisms. In particular, there is a need for rehabilitation programs that allow individuals with MD to continue their daily life activities. Of these daily living activities, reading is often one of the primary rehabilitation goals (Owsley et al., 2009). Because of the significant increase in the prevalence of MD and the importance of reading in daily life, it is important to analyse the factors limiting reading performance in people with central field loss. Namely, a better understanding of these factors will lead to improved methods for reading rehabilitation. The focus of this article is to review and discuss the methods and adaptations available today that we can use in rehabilitation of reading skills in individuals with MD.

\section{Reading skills}

Reading is one of the most important visual functions in daily life. For individuals with MD, reading is usually slow and effortful (Faye, 1976; Fletcher, 1999; Legge et al., 1992). These people experience difficulties in reading due to central-field loss.

Individuals with MD tend to suffer the greatest difficulty with reading because they must rely on their peripheral vision. Depending on the extent of central visual loss, reading with peripheral vision varies from difficult to impossible (Figure 1). Namely, reading speed is dramatically reduced when readers cannot use their central vision.

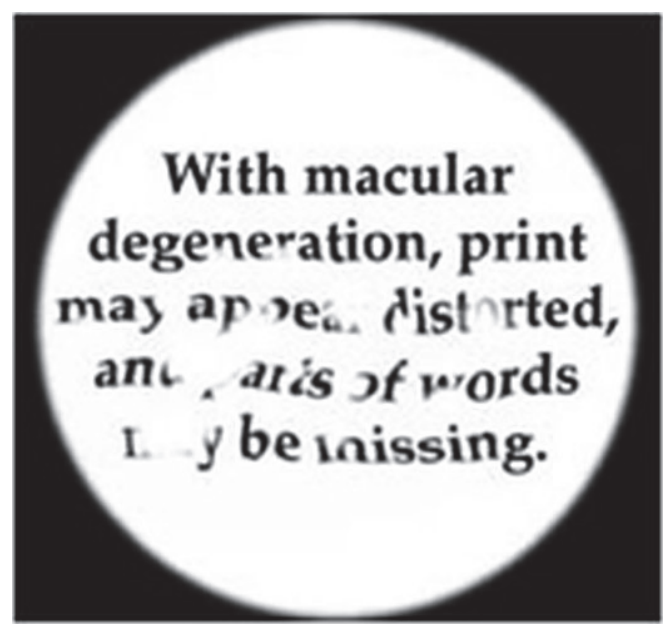

Figure 1. Simulated vision at people with MD 
A central scotoma affects the fovea, which is the centre of the macula, with the highest visual acuity. Visual acuity decreases rapidly as the distance from the fovea increases. Reading with a central scotoma is very difficult, because the scotoma is constantly obscuring the characters to be read. Because people with advanced MD cannot longer use the fovea for reading, they must rely on a peripheral retinal area compensating for the damaged fovea. A major problem is that visual acuity and contrast sensitivity are largely reduced in the peripheral retina (Figure 2). To compensate for this acuity loss, magnification-large print size is required for peripheral reading. However, even with sufficient magnification, peripheral reading remains difficult and slow (Chung, Mansfield, \& Legge, 1998).

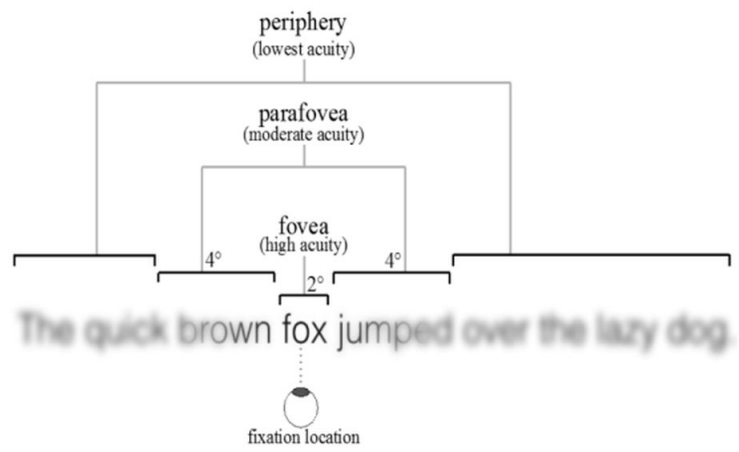

Figure 2. Visual acuity of peripheral vision

Many aspects of reading can be quantified; however, reading speed may be the most representative for reading skills abilities assessment. Reading speed of patients with central scotomas can be 20 to 50 words per minute (wpm) or fewer (Cummings, 1985) or from one fifth to one third the rate of normally sighted subjects (Legge \& Glenn, 1991). Despite the presence of a central scotoma, some persons with MD can handle their disability fairly well if eccentric fixation is established. The use of the eccentric viewing technique can develop spontaneously within 6 months of disease onset, and has been associated with improvements in reading speed.

The region around the point of fixation within which a letter can be resolved, termed the visual span, has long been recognized as an important factor in determining reading speed. Legge et al. (2001) reported visual spans of less than two letters in peripheral vision, compared with ten letters in central vision, and concluded that reduced visual span was a major factor limiting peripheral reading speeds. Even when appropriately compensated for differences in visual acuity, visual span appears to be smaller in peripheral vision, which has an adverse effect on letter and word recognition. Reduction of the size of the visual span is another possible explanation for slow reading performance in MD persons. 
Another factor that is limiting reading rate in peripheral reading is poor oculomotor control. There is evidence of a link between the reading rate and the stability of fixation in the MD. Fixation stability refers to the ability to maintain a visual image in a discrete and stable retinal area during fixateon: the larger that retinal area, the more unstable fixation. Training to achieve better control of fixation is part of many rehabilitation programs for people with macular scotomas. Warren's workbook exercises can be used for training to increase speed and fixation stability. A first exercise contains 2 vertical columns of letters that are situated on opposite ends of the page. The patient with MD starts reading aloud the first letter in the left column and then reads the first letter in the right column, continuing to the second letter of the left column, followed by the second letter of the right column. The patient continues to read the letters in each column until the bottom of the page is reached. The second exercise contains several rows of random letters. In each row, 2 or 3 letters are underlined. The patient is instructed to read aloud only the underlined letters.

In summary, oculomotor deficits play a large role in reducing reading skills after macular function loss. Methods for training of reading skills without usage of eye movements may remove one of the limiting factors. A technique called the 'steady-eye' strategy, where the reader holding a steady eccentric viewing position while moving the page of text from right-to-left in front of their eyes.

In the presence of a bilateral central scotoma, many individuals with MD develop an eccentric preferred retinal area to substitute for the function of the fovea (Fletcher \& Schuchard, 1997). This area is called the Preferred Retinal Locus (PRL). The PRL may be located vertically (above or below) relative to the scotoma or lateral (left or right) to the scotoma (Calabrèse, Liu \& Legge, 2017). A majority of individuals with MD adopt a lateral PRL, and more specifically, a left-field PRL (Schoessow \& Fletcher, 2012). However, research on reading and eye movements in subjects with normal vision indicates that more information about word identity is obtained from letters to the right of fixation. According to one estimate, the normal reader acquires letter shape information from 12-15 letters in the right half of the field, but only 4-5 letters in the left half. Thus, it would seem better to use a PRL to the right of a scotoma instead of to the left (Rubin, 2001). The people with PRLs lateral to a scotoma to have a smaller horizontal visual span, and as a result, to experience slower reading speed with horizontal text. Additionally, left PRLs are expected to be more problematic when is reading from left to right because the scotoma occludes the upcoming words. Therefore, in some cases, it is beneficial to use a vertically oriented text to avoid the upcoming text by the scotoma and would afford the possibility of a larger visual span.

Visual crowding may be one of the most significant factors that limit the ability to read eccentrically-viewed text. Crowding refers to the phenolmenon with which visual stimuli become harder to identify when flanked by other objects. But, a recent study has provided some intriguing results. Nonetheless, even with exaggerated word and line spacing, improvements in reading speed tend to be modest for individuals with moderate to severe 
central visual loss. Namely, several experiments that reduced letter crowding have shown a null or modest effect on reading speed improvement. Chung et al. (2002) added extra-spaces between letters to reduce letter crowding, without any significant improvement for central and peripheral reading speed in normally-sighted readers and patients with central field loss. Adding extra spaces between words (increasing interline and/or inter-word spaces) was also shown to have a modest effect on reading speed performance.

\section{Fonts Designed for Macular Degeneration}

There is evidence showing that alternating contrast polarity of letters can improve letter recognition in peripheral vision (Chung \& Mansfield, 2009). Moreover, few studies have focused specifically on fonts for reading with MD. Mansfield et al. (1996) showed that patients with central vision loss read faster and at a smaller print size when text was printed in Courier rather than in Times Roman. Several fonts (e.g., APHont, Tiresias) have been designed and recommended exclusively for low-vision readers, but there is no clear evidence showing advantages of these special fonts over mainstream fonts for individuals with MD. Two new fonts, Eido and Maxular Rx, both illustrated in Figure 3, were recently designed particularly for individuals with MD.

Helvetica

All the cousins had

a glass of milk and

a bowl of ice cream
Times-Roman

All the cousins had

a glass of milk and

a bowl of ice cream
Courier

All the cousins had

a glass of milk and

a bowl of ice cream

Eido

Maxular

All The cousins had

All the cousins had

A glass of milk and

a glass of milk and

A bowl of icE cream

a bowl of ice cream

Figure 3. Eido and Maxular Rx fonts

The letter templates of the Eido font were inspired by the letter templates of the DejaVu font, similar to Courier, but a smaller complexity. The Eido font letters have been designed following three main principles: Reducing physical similarities between letters, Reducing letter visual complexity and Designing letters that observers are familiar with (Bernard, Aguilar \& Castet, 2016). Maxular Rx was designed to be very bold, have an extreme $x-$ height, and eliminate sharp angles. This font uses extra spacing between letters and between lines to reduce visual crowding. Slight distortions of pro- 
portion were used in the "a," "e," and "s" to increase the recognition of the counters (the internal spaces of those letters).

\section{Illumination}

The effect of illumination on visual performance of low vision subjects has been the topic of many studies over the past 30 years. There is general consensus that the majority of low vision subjects show an improvement in visual acuity with increasing illumination. One question of relevance to low vision practitioners is the extent to which reading performance can be improved in persons with MD through the provision of additional lighting. In particular, there are some subjects with macular disease who demonstrate very significant increases in visual acuity with increased illumination. However, it should be noted that the optimal level of illumination varies among individuals. Elclred (1992) found optimal levels for a group of MD subjects in the range 500 to 7,500 lux. This range included the lowest and highest illuminance levels tested in the study. Therefore, for some MD patients, the optimal level might have been below 500 lux, or above 7,500 lux. Proper illumination of reading materials is also essential. In general, the light source illumination the reading material should be positioned above or behind the person, on the side of the better eye that does the reading.

\section{Conclusion}

Since no effective treatment is available to restore normal central vision, it is essential to optimize the capabilities of peripheral vision for reading. The eccentric viewing and steady-eye techniques can be combined and there is some evidence that these strategies reduce reading difficulties. Hence before implementation of any rehabilitation program for reading skills it is essential to assess PRL location and characteristics.

In many cases, additional training devices are required for optimal reading skills rehabilitation results. These include the provision of optical or electronic magnifying devices, contrast enhancement and use of other nonoptical methods such as supplementary lighting. For purposes of training reading skills, one has to use appropriate training materials. Namely, it is recommended to use print material at $100 \%$ contrast black on white. Also, consider that font has an effect on the reading performance of patients with MD. Two new fonts, Eido and Maxular Rx were found the most advantageous font for persons with MD, and Arial is the worst for reading. 


\section{References}

Bernard, J. B., Aguilar, C., and Castet, E. (2016). A new font, specifically designed for peripheral vision, improves peripheral letter and word recognition, but not eye-mediated reading performance. PLoS One, 11, pp. 1-25.

Calabrèse, A., Liu, T., and Legge, G. E. (2017). Does Vertical Reading Help People with Macular Degeneration: An Exploratory Study. PloS one, 12(1), e0170743. doi:10.1371/journal.pone.0170743

Chung, S. T. L. (2002). The effect of letter spacing on reading speed in central and peripheral vision. Invest Ophthalmol Vis Sci., 43, pp. 1270-1276.

Chung, S. T. L., and Mansfield, J. S. (2009). Contrast polarity differences reduce crowding but do not benefit reading performance in peripheral vision. Vision Res., 49, pp. 2782-2789.

Chung, S. T. L., Mansfield, J. S., and Legge, G. E. (1998). Psychophysics of reading. XVIII. The effect of print size on reading speed in normal peripheral vision. Vision Research, 38, pp. 2949-2962.

Cummings, R. W., Whittaker, S. G., Watson, G. R., and Budd, J. M. (1985). Scanning characters and reading with a central scotoma. Am J Optom Physiol Opt., 62, pp. 833-843.

Faye, E. E. (1976). Clinical Low-Vision. Boston, MA: Little, Brown.

Fletcher, D. C. and Schuchard, R. A. (1997). Preferred retinal loci relationship to macular scotomas in a low-vision population. Ophthalmology, 104, pp. 632-638.

Fletcher, D. C., Schuchard, R.A., and Watson, G. (1999). Relative locations of macular scotomas near the PRL: effect on low vision reading. J Rehabil Res Dev., 36, pp. 356-364.

Jager, R. D., Mieler, W. F., and Miller, J. W. (2008). Age-related macular degeneration. The New England Journal of Medicine, 358 (24), pp. 26062617.

Klein, R., Klein, B .E., Jensen, S.C., and Meuer, S.M. (1997). The five-year incidence and progression of age-related maculopathy: the Beaver Dam Eye Study. Ophthalmology, 104, pp. 7-21.

Legge, G. E, Ross, J. A., Isenberg, L.M., and LaMay, J. M. (1992). Psychophysics of reading: clinical predictors of low-vision reading speed. Invest Ophthalmol Vis Sci., 33, pp. 677-687.

Legge, G. E., and Glenn, A. (1991). Fry Award Lecture 1990: three perspectives on low vision reading. Optom Vis Sci., 68, pp. 763-769. 
Mansfield, J. S., Legge, G. E, and Bane, M. C. (1996). Psychophysics of reading. XV. Font effects in normal and low vision. Invest Ophthalmol Vis Sci., 37, pp. 1492-1501.

Owsley, C., McGwin Jr, G., Lee, P. P., Wasserman, N., and Searcey, K. (2009). Characteristics of low-vision rehabilitation services in the United States. Archives of Ophthalmology, 127(5), pp. 681-689.

Rubin, G. S. (2001). Vision rehabilitation for patients with age-related macular degeneration. Eye, 15(3), pp. 430-435.

Schoessow, K., and Fletcher D. C. (2012). Preferred Retinal Loci Relationship to Macular Scotomas: A 10-Year Comparison. Journal of Visual Impairment \& Blindness, 106, pp. 745-750

Wong W. L., Su X., Li X., Cheung C. M. G., Klein R., Cheng C.-Y., et al. (2014). Global prevalence of age-related macular degeneration and disease burden projection for 2020 and 2040: a systematic review and meta-analysis. Lancet Glob Health 2, e106-16. 10.1016/S2214-109X(13) 70145-1 